\title{
Analysis of ocean in situ observations and web-based visualization
}

Alexander Barth (1), Sylvain Watelet (1), Charles Troupin (2), Aida Alvera Azcarate (1), Giorgio Santinelli (3), Gerrit Hendriksen (3), Alessandra Giorgetti (4), and Jean-Marie Beckers (1)

(1) University of Liege, AGO/GHER, Liege, Belgium (a.barth@ulg.ac.be), (2) Balearic Islands Coastal Ocean Observing and Forecasting System (SOCIB), Palma de Mallorca, Spain, (3) Deltares, Delft, Netherlands, (4) OGS, Istituto Nazionale di Oceanografia e di Geofisica Sperimentale, Trieste, Italy

The sparsity of observations poses a challenge common to various ocean science disciplines. Even for physical parameters where the spatial and temporal coverage is higher, current observational networks undersample a broad spectrum of scales. The situation is generally more severe for chemical and biological parameters because related sensors are less widely deployed.

The analysis tool DIVA (Data-Interpolating Variational Analysis) is designed to generate gridded fields from in situ observations. DIVA has been applied to various physical (temperature and salinity), chemical (concentration of nitrate, nitrite and phosphate) and biological parameters (abundance of a species) in the context of different European projects (SeaDataNet, EMODnet Chemistry and EMODnet Biology). We show the technologies used to visualize the gridded fields based on the Web Map Services standard. Visualization of analyses from in situ observations provides a unique set of challenges since the accuracy of the analysed field is not spatially uniform as it strongly depends on the observations location. In addition, an adequate handling of depth and time dimensions is essential. Beside visualizing the gridded fields, access is also given to the underlying observations. It is thus also possible to view more detailed information about the variability of the observations. The in situ observation visualization service allows one to display vertical profiles and time series and it is built upon OGC standards (the Web Feature Service and Web Processing Services) and following recommendation from the INSPIRE directive. 